\title{
Analysis of Adjustable and Fixed DRX Mechanism for Power Saving in LTE/LTE-Advanced
}

\author{
Scott Fowler \\ Mobile Telecommunications \\ Dept. of Science and Tech. \\ Linköping Univ., Norrköping, Sweden
}

\author{
Ranjeet S. Bhamber \\ Instituto de Óptica "Daza de Valds" \\ C.S.I.C. 121, Serrano, \\ 28006 Madrid, Spain
}

\author{
Abdelhamid Mellouk \\ LiSSi laboratory \\ Dept. of Networks and Telecoms, IUT C/V \\ University of Paris-Est Creteil (UPEC), France
}

\begin{abstract}
The 4G standard Long Term Evolution (LTE) has been developed for high-bandwidth mobile access for today's data-heavy applications, consequently, a better experience for the end user. To extend the user equipment battery lifetime, plus further support various services and large amount of data transmissions, the 3GPP standards for LTE/LTE-Advanced has adopted discontinuous reception (DRX). However, there is a need to optimize the DRX parameters, so as to maximize power saving without incurring network re-entry and packet delays. In this paper, we take an overview of the fixed frame DRX cycle and compare it against an adjustable DRX cycle of the LTE/LTEAdvanced power saving mechanism, by modelling the system with bursty packet data traffic using a semi-Markov process. Based on the analytical model, we will show the trade-off relationship between the power saving and wake-up delay performance.
\end{abstract}

Index Terms-Broad band networks, quality of service, WDM.

\section{INTRODUCTION}

Today, we are starting to see a variety of powerful smart mobile devises (e.g. iPhone, iPad, Android) handling a wide range of traffic including multimedia. However, the current 3G (third generation) wireless cellular technology has been unsuccessful in delivering multimedia with an acceptable level of quality due to the low transmission rate and high service costs. Thus, a 4G (fourth generation) standard, LTE/LTEAdvanced has been developed that is intended for larger capacity and higher speed of mobile networks.

While 4G LTE/LTE-Advanced increases data rates by a factor of 50 over $3 \mathrm{G}$ networks, the battery, the power source of mobile devices, have not seen any sizeable advancement and still possess the same energy density characteristics. Thus substantial improvements in energy-efficient operation mechanisms are necessary for accommodating these very high data rates in 4G LTE/LTE-Advanced (henceforth referred to as LTE) [5].

Through monitoring the activities of the User Equipment (UE), Discontinuous Reception (DRX) is able to lengthen the battery life. The objective of such mechanisms is to turn off the radio signal (or sleep) for the maximum length of time, while staying connected to the network, thus reducing energy consumption when there is no data transmission. Hence, the receiver should be operated discontinuously for downlink (DL) services. In fact, DRX is not a novel idea in LTE [2] since it has been applied in the $2^{\text {nd }}$ generation system, e.g. the Global System for Mobile Communications (GSM). In [7] models the LTE DRX and proves that the LTE DRX achieves a more power saving gain over Universal Mobile Telecommunications
System (UMTS) DRX [1] at the cost of prolonged wake up delay (The same can be said about GSM).

The main difference between LTE and previous DRX is whether the UE is allowed to enter a sleep state when the traffic buffer is not empty. The change of states in LTE DRX relies heavily on scheduling since it will lengthen the active time of an UE by restarting the Inactivity Timer. Meanwhile, due to the sleep duration, the scheduling in LTE DRX is affected by the DRX accordingly. The theoretical basis of traditional scheduling mechanisms becomes invalid when DRX is adopted. The present LTE DRX is based on static sleep mode, consequently performance degradation is inevitable. To address this problem there is a need to optimize the DRX parameters, so as to maximize power saving without incurring network re-entry and packet delays. In particular, care should be exercised for real-time services. In this paper we investigate the use of adjustable and non-adjustable DRX cycle frame duration in LTE.

\section{LTE AND THE DRX CONCEPT}

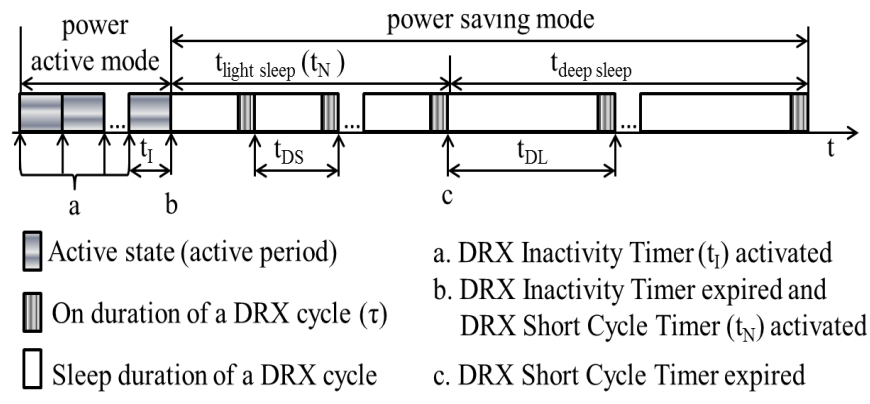

Figure 1: LTE DRX timing for UE receiver operations.

It is particularly important for mobile communications to have efficient power saving mechanisms as the energy source for wireless devises is limited. Therefore, LTEs proposed method for energy efficient operation is to utilize sleep $(\mathrm{OFF}) /$ wake $(\mathrm{ON})$ scheduling.

LTEs energy efficient strategy exploits the concepts of DRX and Discontinuous Transmission (DTX) [3]. By using DRX/DTX, the terminal can turn the radio frequency modem into sleep mode for prolonged period either in RRC_IDLE or RRC_CONNECTED state.

In the LTE DRX mechanism, the sleep/wake scheduling of each UE is determined by the following four parameters [3]: DRX Short Cycle $\left(t_{D S}\right)$, DRX Long Cycle $\left(t_{D L}\right)$, DRX Inactivity Timer $\left(t_{I}\right)$ and DRX Short Cycle Timer $\left(t_{N}\right)$ as 
shown in Figure 1. The $t_{D S}$ and $t_{D L}$ define duration of OFF and ON period, which is a fixed value applied to both long and short cycles. UE monitors the physical downlink control channel $(\mathrm{PDCCH})$ to determine if there is any transmission over the shared data channel allocated to the UE during ON duration. The $t_{I}$ specify the period where UE should stay awake and monitor PDCCH after the last successful decoding of PDCCH. The $t_{N}$ specifies the period where UE should follow $t_{D S}$ after the $t_{I}$ has expired.

In LTE DRX, the sleep/wake-up mode consists of the three different states, namely, Inactivity period, Light Sleep period, and Deep Sleep period. The Inactivity period is the power active mode, whereas the Light Sleep period and the Deep Sleep period are the power saving mode. The transition from the Inactivity period to the Light Sleep period is controlled by $t_{I}$, while the transition from the Light Sleep period to the Deep Sleep period within the power saving mode is controlled by $t_{N}$.

The following describes how the UE receiver works during the Inactivity period, Light Sleep period, and Deep Sleep period [2].

DRX Inactivity period: When the DRX Inactivity Timer ${ }^{1}$ is $\mathrm{ON}$ and the UE receiver is monitoring the $\mathrm{PDCCH}$, at the same time ready to receive packets through the evolved node-B (eNB) from Evolved Packet Core (EPC). The DRX Inactivity Timer, (when not time out) the PDCCH indicated a Downlink transmission or Uplink transmission. Should the DRX Inactivity Timer expire, then the DRX Short Cycle Timer is activated and the Light Sleep period begins.

DRX Light Sleep period: The period is the DRX Short Cycle $\left(t_{D S}\right)$. During each of the DRX Short Cycle the UE wakes up to monitor the PDCCH (Active state (active period) or also know as Listen Interval in Figure 1). If the PDCCH indicates a downlink transmission, the UE change from Light Sleep period to an activity period and starts the DRX Inactivity Timer. Otherwise the UE will return to Light Sleep period. The UE will keep entering Light Sleep period until the DRX Short Cycle Timer ${ }^{2}$ expires. While in DRX Light Sleep period the eNB will not transmit any packets to the UE.

DRX Deep Sleep period: During each of the DRX Deep Long Cycle the UE wakes up to monitor the PDCCH. If the PDCCH indicates a downlink transmission, the UE changes from Deep Sleep period to activity period and starts the DRX Inactivity Timer. Otherwise the UE will return to Deep Sleep period. While in DRX Deep Sleep period the eNB will not transmit any packets to the UE.

\section{An Analytical Model for LTE Power Saving}

\section{A. Bursty Packet Traffic Model}

Studies have shown that for some environments, the traffic data are self-similar [13] rather than the traditional queuing

\footnotetext{
${ }^{1}$ Inactivity Timer: Specifies the number of consecutive TTIs during which UE shall monitor PDCCH after successfully decoding a PDCCH indicating a UL or DL data transfer for this UE.

${ }^{2}$ DRX Short Cycle Timer $\left(t_{N}\right)$ : Indicates the number of initial DRX cycles to follow the short DRX cycle before transitioning to the long DRX cycle.
}

that is contingent on the data traffic to be Poisson. In the traditional Poisson Traffic model, it usually has a very limited range of time scales, making it short range dependent. With self-similar traffic, it displays burstiness and interacts over an immensely wide range of time scales, making it long range dependent. In addition, it has been shown to be heavy tailed such as Pareto and Weibull distributions are more applicable when modeling data network traffic [9]. For this paper, we used the European Telecommunication Standards Institute (ETSI) traffic model [4], where the packets size and the packet transmission timer are assumed to follow the truncated Pareto distribution. The [4] is a widely used in various analytical and simulation studies of 3GPP networks, such as [7], [10], [12], [16], [17].

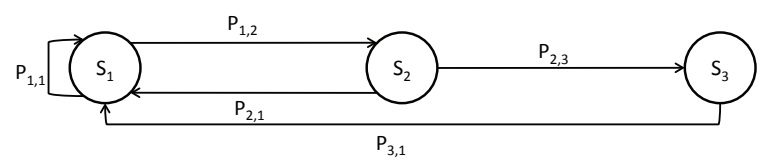

Figure 2: A semi-Markov process for LTE DRX analysis.

The LTE DRX mechanism is a semi-Markov process [11] and is illustrated in Figure 2. The state transition diagram consists of three states, which are relevant to the three periods show in Figure 1.

- State $S_{1}$ comprises a sequence of adjacent active time intervals corresponding to the entire duration of a single packet call transmission, i.e. the UE is in power active mode.

- State $S_{2}$ comprises a Light Sleep period $\left(t_{\text {light sleep }}\left(t_{N}\right)\right)$ which is entered from $S_{1}$, i.e. the UE follows DRX Short Cycles.

- State $S_{3}$ comprises a Deep Sleep period ( $t_{\text {deep }}$ sleep $)$ which is entered from $S_{2}$, i.e. the UE follows DRX Long Cycles.

A new packet call can be viewed as continuation of the current session (Condition 1) or as the onset of a new session (Condition 2) depending on the time interval-arrive between two consecutive packet calls. The packet calls may be the inter-packet call idle time $\left(t_{i p c}\right)$ with probability $P_{p c}=1$ $1 / \mu_{p c}$ or the inter-session idle time $\left(t_{i s}\right)$ with probability $P_{s}$ $=1 / \mu_{p c}$. The probabilities take into account the memoryless property of a geometric distributions.

If we view this semi-Markov process only at the times of state transitions, we obtain an embedded Markov chain with state transition probabilities $P_{i, j}$, where $i, j \in\{1,2,3\}$. Next, we derive these state transition probabilities.

\section{B. State 1 to State 1 and State 1 to State 2}

State $S_{1}$ contains $N_{p}$ inactivity periods ${ }^{3}$. During the last inactivity period, if the PDCCH indicates the next packet call delivery happened before DRX Inactivity Timer expires, the DRX Inactivity Timer is cancelled, another inactivity period is started and state $S_{1}$ is re-entered $\left(t_{I}\right.$ has not expired); otherwise, state $S_{2}$ is entered when DRX Inactivity Timer expires. The probability that a new packet call begins before

\footnotetext{
${ }^{3} N_{p}$ : Number of packets per packet call.
} 
the expiration of $t_{I}$ is $q_{1}=\operatorname{Pr}\left[t_{i p c}<t_{I}\right]=1-\mathrm{e}^{-\lambda_{i p c} t_{I}}$ in Condition 1 and $q_{2}=\operatorname{Pr}\left[t_{i s}<t_{I}\right]=1-\mathrm{e}^{-\lambda_{i s} t_{I}}$ in Condition 2 , respectively. Then we have:

$$
\begin{gathered}
P_{1,1}=P_{p c} q_{1}+P_{s} q_{2} \\
P_{1,2}=P_{p c}\left(1-q_{1}\right)+P_{s}\left(1-q_{2}\right)
\end{gathered}
$$

\section{Adjustable DRX Timer State for Light Sleep}

Short DRX Cycle is the first DRX cycle to be followed after enabling DRX. The probable short DRX cycles are $2^{n}$, where $n=1,2, \ldots 9$ and $5 \times 2^{m}$, where $m=1,2, \ldots 6$ in terms of subframes or time. The duration of the $n^{\text {th }}$ sleep interval is obtained by:

$$
T(n)= \begin{cases}\kappa 2^{n} & 1 \leq \mathrm{n}<\mathrm{M} \\ T_{\max } & \mathrm{M} \geq \mathrm{n}\end{cases}
$$

where $M$ is the value that $T(n)=T_{\max }$ and $\kappa$ is a rescaling factor, which is used to control the total Light Sleep cycle duration. The duration of the $k^{t h}$ sleep cycle, which consists of a sleep interval and a listening, is given by:

$$
C_{D S}^{n}=T_{n}+L
$$

where $L$ is the duration of listen interval.

The probability that there is no initiation of awakening during $C_{D S}^{n}\left(P_{n}\right)$ is obtained by:

$$
P_{n}=e^{-\lambda C_{D S}^{n}}, 1 \leq \mathrm{n}<\mathrm{M}
$$

According to the Figure 1, the transition from Light Sleep to Deep Sleep consist of:

$$
t_{C S}=t_{N}=\kappa \sum_{1}^{n} C_{D S}^{n}
$$

\section{State 2 to State 1 and State 2 to State 3}

According to adjustable DRX Light Sleep cycle, the probability that there is at least one initiation of awakening during $C_{D S}$ is $1-\mathrm{e}^{-\lambda C_{D S}}$. In state $S_{2}$ the UE follows DRX Short Cycles. If the PDCCH indicates that a new packet call starts before the adjustable DRX Short Cycle Timer expires (means new packet call occurs before $t_{N}$ has expired), the timer is cancelled, and state $S_{1}$ is entered; otherwise state $S_{3}$ is entered. The probability that there is at least one initiation of awakening in the $n^{\text {th }}$ sleep cycle during a sleep-mode operation is achieved by:

$$
\begin{aligned}
P_{2,1} & =P_{p c} \cdot \sum_{\alpha}^{n}\left(1-P_{\alpha}\right)+P_{s} \cdot \sum_{\alpha}^{n}\left(1-P_{\alpha}\right) \\
& =P_{p c}\left(1-\prod_{\alpha}^{n} e^{-\kappa \lambda_{i p c} C_{D S}^{\alpha}}\right)+P_{s}\left(1-\prod_{\alpha}^{n} e^{-\kappa \lambda_{i s} C_{D S}^{\alpha}}\right)
\end{aligned}
$$

and

$$
\begin{aligned}
P_{2,3} & =P_{p c} \cdot \sum_{\alpha}^{n} P_{\alpha}+P_{s} \cdot \sum_{\alpha}^{n} P_{\alpha} \\
& =P_{p c} e^{-\lambda_{i p c} \kappa \sum_{\alpha}^{n} C_{D S}^{\alpha}}+P_{s} e^{-\lambda_{i s} \kappa \sum_{\alpha}^{n} C_{D S}^{\alpha}} \\
& =P_{p c} \prod_{\alpha}^{n} e^{-\kappa \lambda_{i p c} C_{D S}^{\alpha}}+P_{s} \prod_{\alpha}^{n} e^{-\kappa \lambda_{i s} C_{D S}^{\alpha}}
\end{aligned}
$$

\footnotetext{
${ }^{4} t_{B}$ : consists of the number of packet within a per packet call $\left(N_{p}\right)$.

${ }^{5} t_{x}$ : The time interval between when the packet is transmitted by the LTE RNC processor and when the corresponding positive ACK is received by the LTE RNC processor.
} thus, we have $P_{3,1}=1$.

\section{F. Transition Probability Matrix}

The transition probability matrix $\mathbf{P}=\left(P_{i, j}\right)$ of the embedded Markov chain can, hence, be given as (8):

$$
\mathbf{P}=\left[\begin{array}{ccc}
P_{1,1} & P_{1,2} & 0 \\
P_{2,1} & 0 & P_{2,3} \\
1 & 0 & 0
\end{array}\right]
$$

Let $\pi_{i}(i \in\{1,2,3\})$ denote the probability that the embedded Markov chain is in state $S_{i}(i \in\{1,2,3\})$. By using $\sum_{j=1}^{3} \pi_{i}=1$ and the balance equation $\pi_{i}=\sum_{j=1}^{3} \pi_{j} P_{j, i}$, we can solve the stationary distribution and obtain (9)

$$
\Pi=\left\{\begin{array}{lll}
\pi_{1} & = & \frac{1}{1+P_{1,2}+P_{1,2} P_{2,3}} \\
\pi_{2} & = & \frac{P_{1,2}}{1+P_{1,2}+P_{1,2} P_{2,3}} \\
\pi_{3} & = & \frac{P_{1,2} P_{2,3}}{1+P_{1,2}+P_{1,2} P_{2,3}}
\end{array}\right.
$$

Let $H_{i}(i \in\{1,2,3\})$ represent the holding time of the semiMarkov process at state $S_{i}(i \in\{1,2,3\})$. Now we proceed to derive $E\left[H_{i}\right]$.

$\mathrm{E}\left[H_{i}\right]$ : In state $S_{1}$, mobile device experiences a busy period $t_{B}{ }^{4}$ and then an interpacket call inactivity period $t_{I}$. In LTE 8-process Stop-And-Wait Hybrid Automatic Request (SAWHARQ) flow-control algorithm is implemented in packet transmission, which can be modelled as an M/M/8 queuing model. According to [6] we have:

$$
E\left[H_{1}\right]=E\left[t_{B}\right]+E\left[t_{I}\right]
$$

Since a busy period is identical to the duration of a packets call delivery, a $t_{B}$ consists of $N_{p}$ packets service times $t_{x}{ }^{5}$. From Wald's Theorem 5.18 [8], we have

$$
E\left[t_{B}\right]=E\left[N_{p}\right] E\left[t_{x}\right]=\frac{\mu_{p}}{\lambda_{x}}
$$

where $\mu_{p}$ is the number of packets calls within a packet service session and $\lambda_{x}$ is the Inter-packet arrive time.

If a packets arrives before the Inactivity Timer expires $\left(t_{i p c}<t_{I}\right)$, then the Inactivity period equals the inter-packet call idle time, $t_{I}=t_{i p c}$; Otherwise the next packet arrives after the DRX Inactivity Timer has expired $\left(t_{I} \geq t_{i p c}\right)$. Therefore, we have $t_{I}=\min \left(t_{i p c}, t_{I}\right)$. Similarly, in Inter-session idle time $\left(t_{i s}\right)$, we have $t_{I}=\min \left(t_{i s}, t_{I}\right)$.

Therefore, we have for $t_{I}$ for $t_{i p c}$ and $t_{i s}$ yields:

$$
E\left[t_{I}\right]=P_{p c} E\left[\min \left(t_{i p c}, t_{I}\right)\right]+P_{s} E\left[\min \left(t_{i s}, t_{I}\right)\right]
$$

We obtain that:
There is only one transition out of state $S_{3}$ to the state $S_{1}$, 


$$
\begin{aligned}
E\left[\min \left(t_{i p c}, t_{I}\right)\right] & =\int_{x=0}^{\infty} \operatorname{Pr}\left[\min \left(t_{i p c}, t_{I}\right)>x\right] d x \\
& =\int_{x=0}^{t_{I}} \operatorname{Pr}\left[t_{i p c}>x\right] d x \\
& =\int_{x=0}^{t_{I}} e^{-\lambda_{i p c} x} d x=\left(\frac{1}{\lambda_{i p c}}\right)\left[1-e^{-\lambda_{i p c} t_{I}}\right]
\end{aligned}
$$

where $f\left(t_{i p c}\right)=\lambda_{i p c} e^{-\lambda_{i p c} t_{i p c}}$ is the $P D F$ of the inter-packet call idle time $\mathrm{t}_{i p c}$. Likewise:

$$
E\left[\min \left(t_{i s}, t_{I}\right)\right]=\left(\frac{1}{\lambda_{i s}}\right)\left[1-e^{-\lambda_{i s} t_{I}}\right]
$$

Substitute equation (13) and (14) into (12)

$$
E\left[t_{I}\right]=\left(\frac{P_{p c}}{\lambda_{i p c}}\right)\left[1-e^{-\lambda_{i p c} t_{I}}\right]+\left(\frac{P_{s}}{\lambda_{i s}}\right)\left[1-e^{-\lambda_{i s} t_{I}}\right]
$$

Substitute equation (11) and (15) into (10)

$$
\begin{aligned}
E\left[H_{1}\right] & =\left(\frac{\mu_{p}}{\lambda_{x}}\right)+\left(\frac{1}{\lambda_{i p c}}\right)\left[1-e^{-\lambda_{i p c} t_{I}}\right] \\
& +\left(\frac{1}{\lambda_{i s}}\right)\left[1-e^{-\lambda_{i s} t_{I}}\right]
\end{aligned}
$$

\section{Adjustable DRX Cycles in 3GPP LTE}

Next we analyze the wake-up delay from the DRX. Whether we are in Deep Sleep or Light Sleep a packet call transmission may begin in one of the sleep states. The probability a packet call delivery starts during the $i^{t h}$ DRX Cycle is in a fixed DRX Cycles:

$p_{i}=\left\{\begin{array}{l}\begin{array}{l}P_{p c} e^{-\lambda_{i p c} t_{I}} e^{-\lambda_{i p c}(i-1) t_{D S}}\left(1-e^{-\lambda_{i p c} t_{D S}}\right) \\ \underbrace{+P_{s} e^{-\lambda_{i s} t_{I}} e^{-\lambda_{i s}(i-1) t_{D S}}\left(1-e^{-\lambda_{i s} t_{D S}}\right),}_{1 \leq i \leq N_{D S}}\end{array} \\ \underbrace{\begin{array}{l}P_{p c} e^{-\lambda_{i p c}\left[\left(t_{I}+N_{D S} t_{D S}+\left(i-N_{D S}-1\right) t_{D L}\right]\right.}\left(1-e^{-\lambda_{i p c} t_{D L}}\right) \\ +P_{s} e^{-\lambda_{i s}\left[\left(t_{I}+N_{D S} t_{D S}+\left(i-N_{D S}-1\right) t_{D L}\right]\right.}\left(1-e^{-\lambda_{i s} t_{D L}}\right)\end{array}}_{i \geq N_{D S}}\end{array}\right.$

However, by having adjustable DRX Sleep cycle in $t_{D S}$, equation (17), the probability a packet call delivery starts during the $\mathrm{i}^{\text {th }}$ DRX Cycle is now:

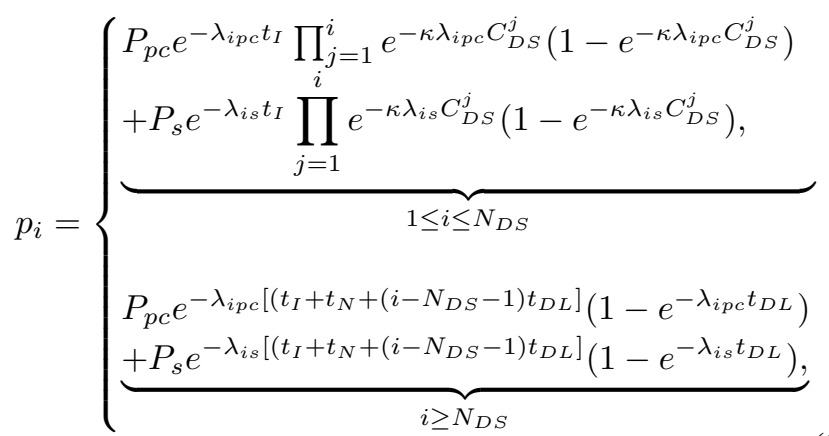

\section{Sleep States $\mathrm{H}_{2}$ AND $\mathrm{H}_{3}$}

State $S_{2}$ comprises a Light Sleep period consisting of $N_{D S}$ DRX Short Cycles. We denote $N_{D S}$ as the total length of $t_{N}$ expressed in terms of the number of DRX Short Cycles. In this case the DRX Short Cycle Timer has expired and state $S_{3}$ is entered. The probability that a new packet call begins before $t_{N}$ expires results in $N_{D S}^{*}$, meaning $N_{D S}^{*}<N_{D S}$. Therefore, the mean holding time in state $S_{2}$ is:

$$
\begin{aligned}
E\left[H_{2}\right] & =E\left[N_{D S}\right]\left\langle t_{C S}\right\rangle \\
& =\left(P_{23} N_{D S}+P_{21} E\left[N_{D S}^{*}\right]\right) \kappa \sum_{i=1}^{n} \frac{C_{D S}^{i}}{n}
\end{aligned}
$$

Due to the memoryless property of the exponential $t_{i p c}$ and $t_{i s}, N_{D S}^{*}$ has a geometric distribution with mean $1 / P_{D S}$, where $P_{D S}$ is the probability that packets arrive during a DRX cycle and is derived as follows:

$$
\begin{aligned}
E\left[N_{D S}^{*}\right] & =\frac{P_{p c}}{\operatorname{Pr}\left[t_{i p c}<C_{D S}\right]}+\frac{P_{s}}{\operatorname{Pr}\left[t_{i s}<C_{D S}\right]} \\
& =\frac{P_{p c}}{1-\prod_{i=1}^{n} e^{-\kappa \lambda_{i p c} C_{D S}^{i}}}+\frac{P_{s}}{1-\prod_{i=1}^{n} e^{-\kappa \lambda_{i s} C_{D S}^{i}}}
\end{aligned}
$$

Then we substitute equations (6), (7) and (20) into (19):

$$
\begin{aligned}
E\left[H_{2}\right] & =\left(\left[P_{p c} \prod_{\alpha}^{n} e^{-\kappa \lambda_{i p c} C_{D S}^{\alpha}}+P_{s} \prod_{\alpha}^{n} e^{-\kappa \lambda_{i s} C_{D S}^{\alpha}}\right] N_{D S}\right. \\
+ & {\left[P_{p c}\left(1-\prod_{\alpha}^{n} e^{-\kappa \lambda_{i p c} C_{D S}^{\alpha}}\right)\right.} \\
+ & \left.P_{s}\left(1-\prod_{\alpha}^{n} e^{-\kappa \lambda_{i s} C_{D S}^{\alpha}}\right)\right] \\
& \cdot\left[\frac{P_{p c}}{1-\prod_{\alpha=1}^{n} e^{-\kappa \lambda_{i p c} C_{D S}^{\alpha}}}\right. \\
+ & \left.\left.\frac{P_{s}}{1-\prod_{\alpha=1}^{n} e^{-\kappa \lambda_{i s} C_{D S}^{\alpha}}}\right]\right) \kappa \sum_{\alpha=1}^{n} \frac{C_{D S}^{\alpha}}{n}
\end{aligned}
$$

State $S_{3}$ contains of Deep Sleep period consisting of State $n_{D L}$ Long DRX Cycles. Therefore:

$$
E\left[H_{3}\right]=\left(\frac{P_{p c}}{1-e^{-\lambda_{i p c} t_{D L}}}+\frac{P_{s}}{1-e^{-\lambda_{i s} t_{D L}}}\right) t_{D L}
$$

\section{POWER SAVING FACTOR (PS)}

The power saving factor (PS) is equal to the probability that the semi-Markov process is at $S_{2}$ and $S_{3}$ in the steady state. Note that each DRX Short Cycle and each DRX Long Cycle contains a fixed On Duration $\tau$ so that it can listen to the paging information from the network. Therefore, the effective sleep duration is $C_{D S}-\tau$ or $t_{D L}-\tau$. Hence, the effective sleep time in both states $S_{2}$ and $S_{3}$ are derived as the following: 


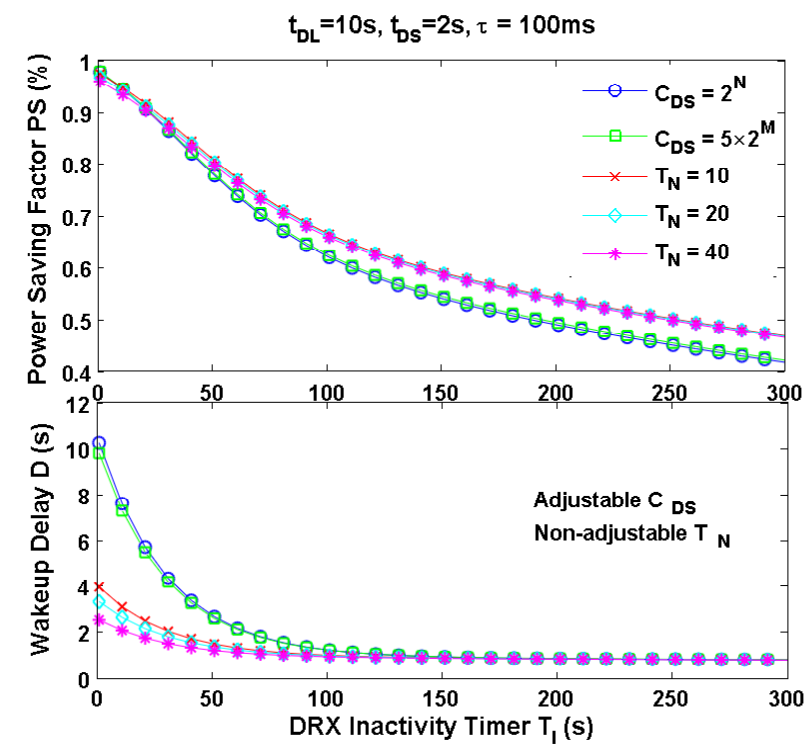

Figure 3: (Top) LTE DRX Inactivity Timer on $T_{I}$ for Power. (Bottom) LTE DRX Inactivity Timer on $T_{I}$ for Delay.

$$
E\left[H_{2}^{\prime}\right]=\left(P_{23} N_{D S}+P_{21} E\left[N_{D S}^{*}\right]\right)\left(\kappa \sum_{i=1}^{n} \frac{C_{D S}^{i}}{n}-\tau\right)
$$

and

$$
E\left[H_{3}^{\prime}\right]=\left(\frac{P_{p c}}{1-e^{-\lambda_{i p c} t_{D L}}}+\frac{P_{s}}{1-e^{-\lambda_{i s} t_{D L}}}\right)\left(t_{D L}-\tau\right)
$$

From Theorem 4.8.3 [11], we obtain $P S=\lim _{t \rightarrow \infty} \operatorname{Pr}$ [UE receiver is turned off at time $t$ ] for $P S$ to be obtain by:

$$
P S=\frac{\pi_{2} E\left[H_{2}^{\prime}\right]+\pi_{3} E\left[H_{3}^{\prime}\right]}{\sum_{i=1}^{3} \pi_{i} E\left[H_{i}\right]}
$$

Substituting Equations (18), (16), (21), (22), (23), (24) into Equation (25), we derive the closed-form equation for the power saving factor PS.

The packet call arrivals follow a Poisson distribution since the inter-packet call idle time and inter-session idle timer are random exponential distributed variables. Also, the arrival event are random observers to the sleep durations [14], [15], [18]. Therefore we have:

$$
E[D]=\sum_{i=1}^{N_{D S}} p_{i} \frac{C_{D S}^{i}}{2 i}+\sum_{i=N+1}^{\infty} p_{i} \frac{t_{D L}}{2}
$$

Substituting Equation (18) into Equation (26), we derive the closed-form equation for the mean of wake-up delay $E[D]$.

\section{NUMERICAL RESULTS}

The effects of the DRX Inactivity Timer $T_{I}$ and the DRX Short Cycle Timer $T_{N}$ are described in Figures 3 - 4. Both $P S$ and $D$ decrease as $T_{I}$ and $T_{N}$ increase with the non-adjustable approach, but this is not always true for the adjustable case.

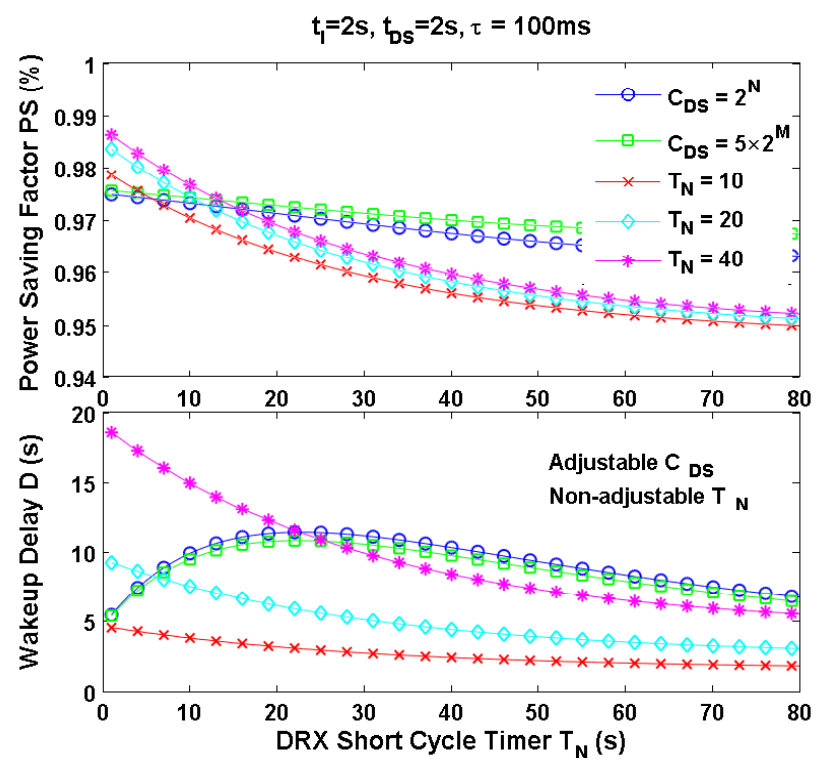

Figure 4: (Top) LTE DRX Short Cycles on $T_{N}$ for Power. (Bottom) LTE DRX Short Cycles on $T_{N}$ for Delay.

When $T_{I}$ becomes larger, in the case of the non-adjustable cycle, it is more probable that a packet call delivery occurs before the DRX Inactivity Timer expires, resulting in fewer transition to the power saving mode. Since the number of transitions to the power saving mode are more infrequent, the impact, of the amount of packet call deliveries delayed, will be minor, consequential both power saving and delay are smaller.

At lower values of $T_{I}$ for the adjustable DRX cycle, the UE resides in the power saving mode longer, however, as $T_{I}$ becomes larger the $P S$ decreases for adjustable DRX while the non-adjustable $P S$ is higher. This is due to less time spent in $P S$ mode, since $C_{D S}$ is smaller with an adjustable DRX at the beginning, but then becomes larger, resulting in fewer transition to the power saving mode.

In Figure 4 the adjustable DRX has a lower power saving value when $T_{N}$ is between $8-18$, but has a greater power saving factor as $T_{N}$ increases. The power saving still decreases, due to the fact that the UE is less likely to enter the Deep Sleep period. Since the adjustable DRX cycle preforms better power saving function at small values of $T_{N}$, the corresponding delay is greater when compared to the non-adjustable case. But as $T_{N}$ increases in size, the delay peaks at around 25 and then decreases, as shown in Figure 4. This behaviour is due to the exponential distribution of the frames within the Light Sleep period and the statistical nature of the packets, which begin to arrive in the more densely packed region of the cycle (.i.e. towards the beginning).

Next we will look at Figures 5 - 6, by focusing on the effects of the DRX Short Cycle $T_{D S}$ and the DRX Long Cycle $T_{D L}$.

The power saving and delay shown in Figure 5 are increasing for both $T_{D S}$ and $T_{D L}$ for the non-adjustable method, which is due to the Sleep Cycles are longer and the "ON Duration is fixed". The longer DRX Cycles translate into more 


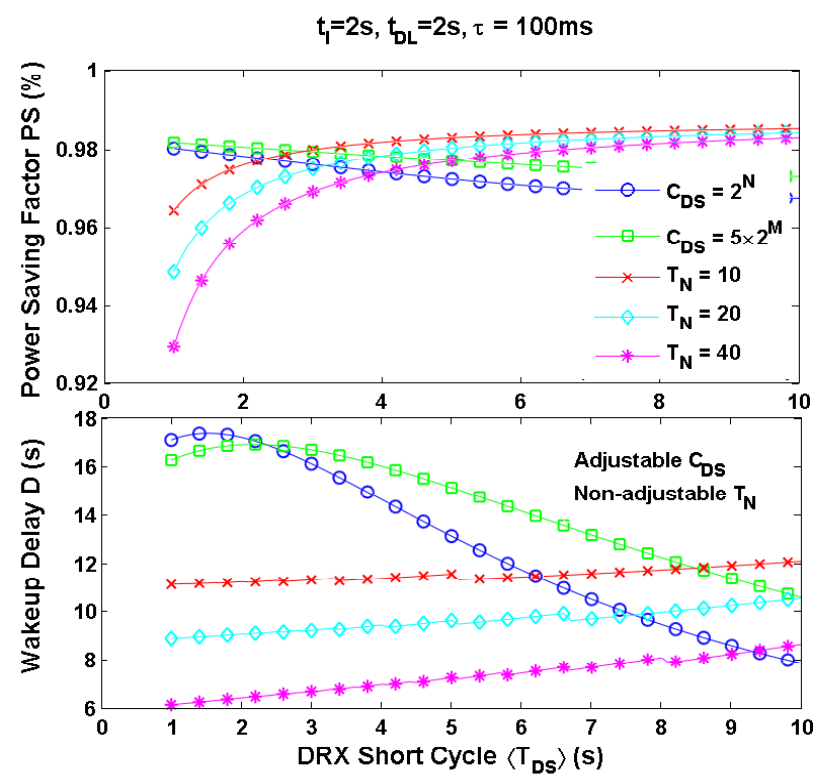

Figure 5: (Top) LTE DRX Short Cycles on $T_{D S}$ for Power. (Bottom) LTE DRX Short Cycles on $T_{D S}$ for Delay.

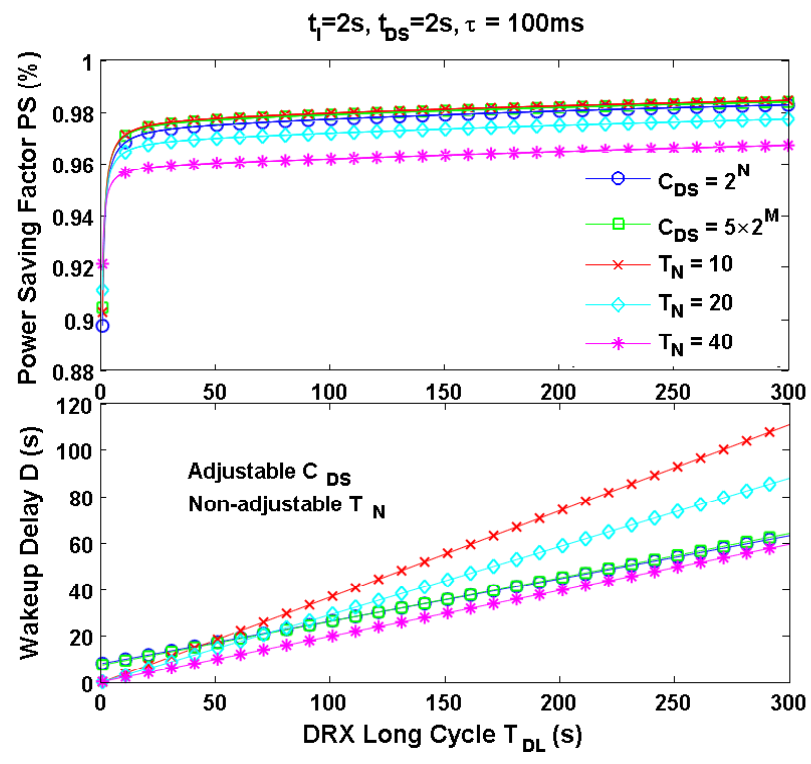

Figure 6: (Top) LTE DRX Long Cycles on $T_{D L}$ for Power. (Bottom) LTE DRX Short Cycles on $T_{D L}$ for Delay.

effective sleep time per cycle, resulting in better power saving and a decrease in performance of the wake-up delay.

The power saving factor in Figure 5 is decreasing for the adjustable case. As the Sleep Cycles are increasing in size and the "ON Duration is adjusting", the longer DRX Cycles translate into less effective sleep time per cycle, resulting in a decrease in performance for power saving. The $T_{D S}$ in Figure 5 has a longer wake-up delay at around 1.5, but then begins to improve, which is due to the correlation between the exponential distributions of the frames within the adjustable DRX cycle and the statistical nature of the packets.

The adjustable DRX cycle is able to adapt to the scheduling behaviour of the system in order to enhancing the power saving gain mechanism for $T_{D L}$. However, having increased the power saving factor, it inevitably effects the performance of the wake-up delay, as in the situation when $T_{N}$ is in the region below and around 30, as shown in Figure 6. Although, at larger values of around 40 and above for both $C_{D S}=2^{N}$ and $C_{D S}=5 \times 2^{M}$ the adjustable DRX cycle has a smaller delay.

\section{CONCLUSION}

In this paper, we have taken an overview of LTE DRX mechanism with adjustable and non-adjustable DRX cycles and model it with bursty packet data traffic using a semiMarkov process. The analytical results show that adjustable LTE DRX will perform differently compare to the nonadjustable LTE DRX. To verify the performance, four DRX parameters on output performance through the analytical model in additional to a trade-off relationship between the power saving and wake-up delay performance was investigated. This work will help to select the best parameters when LTE DRX is implemented.

\section{ACKNOWLEDGMENT}

Scott Fowler was partially supported by the Swedish Excellence Center at Linköping - Lund in information Technology (ELLIIT) and EC-FP7 Marie Curie CIG grant, Proposal number: 294182. Ranjeet S. Bhamber wishes to thank the financial support of Ministerio de Ciencia e Innovación (MICINN) through grant TEC2008-05791.

\section{REFERENCES}

[1] 3GPP TS 25.304. UE procedures in idle mode and procedures for cell reselection in connected mode, v3.14.0, (Release 8), March 2004

[2] 3GPP TS 36.321. Medium Access Control (MAC) protocol specification, v10.2.0, (Release 10), March 2011.

[3] C. Bontu and E. Illidge. Drx mechanism for power saving in lte. Communications Magazine, IEEE, 47(6):48-55, 2009.

[4] ETSI. Universal mobile telecommunications system (umts); selection procedures for the choice of radio transmission technologies of the umts. Technical Report UMTS 30.03, version 3.2.0, April 1998.

[5] S. Fowler. Study on power saving based on radio frame in lte wireless communication system using drx. IEEE Globecom Workshop, December 2011.

[6] Leonard Kleinrock. Queueing Systems: Volume I Theory. Wiley Interscience, New York, Inc., 1975.

[7] Lei Zhou, et al. Performance analysis of power saving mechanism with adjustable drx cycles in 3gpp lte. IEEE 68th Vehicular Technology Conference (VTC 2008), pages 1-5, September 2008.

[8] Randolph Nelson. Probability, stochastic processes, and queueing theory: the mathematics of computer performance modeling. SpringerVerlag New York, Inc., New York, NY, USA, 1995.

[9] V. Paxson and S. Floyd. Wide-area traffic: the failure of poisson modeling. SIGCOMM Comput. Commun. Rev., 24:257-268, October 1994.

[10] R. Mullner, et al. Contrasting open-loop and closed-loop power control performance in utran lte uplink by ue trace analysis. IEEE International Conference on Communications, (ICC '09), pages 1-6, June 2009.

[11] S. M. Ross. Stochastic Processes, 2nd Edition. John Wiley \& Sons, 1996.

[12] Shun-Ren Yang, et al. Modeling umts power saving with bursty packet data traffic. IEEE Transactions on Mobile Computing, 6(12):1398-1409, December 2007.

[13] Walter Willinger, et al. Self-similarity through high-variability: Statistical analysis of ethernet lan traffic at the source level. IEEE/ACM Transactions on Networking, 5(1):71-86, 1997.

[14] Y. Xiao. Energy saving mechanism in the ieee 802.16e wireless man. Communications Letters, IEEE, 9(7):595 - 597, 2005. 
[15] Yang Xiao. Performance analysis of an energy saving mechanism in the ieee 802.16e wireless man. Consumer Communications and Networking Conference, 2006. CCNC 2006. 3rd IEEE, 1:406 - 410, 2006.

[16] Yuanye Wang, et al. Fixed frequency reuse for lte-advanced systems in local area scenarios. Vehicular Technology Conference, 2009. VTC Spring 2009. IEEE 69th, pages 1-5, April 2009.

[17] Yuanye Wang, et al. An interference aware dynamic spectrum sharing algorithm for local area lte-advanced networks. IEEE 70th Vehicular Technology Conference (VTC 2009), pages 1-5, September 2009.

[18] Yan Zhang, Yang Xiao, and V.C.M. Leung. Energy management analysis and enhancement in ieee 802.16e wirelessman. IEEE Transactions on Vehicular Technology, 58(7):3738-3752, September 2009. 